\title{
Stadtluft wird sauberer, Kinder atmen auf
}

\author{
Einige Industrieländer schaffen es inzwischen, die Luftverschmutzung wieder zu verringern. \\ Das schlägt sich direkt in den Lungenfunktionswerten der dort lebenden Kinder nieder.
}

\begin{abstract}
_ Auch wenn Umweltverschmutzung global weiter zunimmt, steigt in einigen westlichen Industrieländern seit Jahren die Luftqualiät. In einer kalifornischen Studie wurden insgesamt 2.120 Kinder standardisierten Lungenfunktionsuntersuchungen unterzogen. Die Kinder teilten sich auf drei Kohorten auf, die in den Jahren 1994-1998, 1997-2001 und 20072011 untersucht wurden. Zu Beginn jeder Periode waren die Probanden 11 Jahre alt.

Mittels linearer Regression wurde untersucht, ob das forcierte Expirationsvolumen in einer Sekunde $\left(\mathrm{FEV}_{1}\right)$ und die forcierte Vitalkapazität (FVC) mit verschiedenen Messgrößen für die Umweltbelastung korrelierten, nämlich den Konzentrationen von $\mathrm{NO}_{2}$, Ozon sowie Feinstaub mit einer Partikelgröße von $<10 \mu \mathrm{m}$ und $<2,5 \mu \mathrm{m}$. Die größeren Partikel dringen beim Menschen bis in die
\end{abstract}

Nasenhöhle vor, die kleineren bis in die Bronchien und Alveolen.

Über die gesamten 13 Jahre ergab sich eine signifikante Zunahme von $\mathrm{FEV}_{1}$ und FVC, die mit der Abnahme des $\mathrm{NO}_{2}$-Gehalts in der Luft korrelierte ( $\mathrm{p}<$ 0,001 für beide Lungenparameter). Gleiches galt für die größeren Luftpartikel ( $p<0,001$ für beide) wie auch für die kleineren ( $\mathrm{p}<0,001$ für die FVC, $\mathrm{p}=$ 0,008 für die $\left.\mathrm{FEV}_{1}\right)$. Der Ozongehalt in der Luft hatte keinen Einfluss auf die Lungenfunktionsparameter.

Das Geschlecht der Kinder sowie das Vorliegen eines Asthma bronchiale hatten keinen Einfluss. Der Anteil der Kinder mit einer klinisch signifikanten Verminderung der $\mathrm{FEV}_{1}(<80 \%$ des Sollwerts) nahm über die drei Kohorten hinweg von $7,9 \%$ auf $6,3 \%$ und $3,6 \%$ in $\mathrm{Ab}$ hängigkeit vom Grad der Luftverschmutzung ab.
- Gauderman WJ, Urman R, Avol Eet al. Association auf improved air quality with lung development in children. NEnglJ Med. 2015;372:905-13

\section{KOMMENTAR}

Die Daten aus Südkalifornien sind sicher auf andere Staaten übertragbar, die in den letzten Jahren die Schadstoffbelastung ihrer Luft gesenkt haben. Sie belegen eindrucksvoll, welche Konsequenz die Luftverschmutzung auf die Entwicklung der kindlichen Lunge hat. Rechnet man diese während eines kurzen Lebensabschnitts erhobenen Daten auf die lebenslange Exposition hoch, wird gut deutlich, welchen Einfluss die Schadstoffbelastung auf die Gesundheit hat. Und dabei sind die verheerenden Folgen des Klimawandels noch gar nicht berücksichtigt.

Die Studie liefert einen Grund mehr, alle Anstrengungen zu unternehmen, durch die Verbesserung der Umweltqualität die Lebensqualität des Menschen zu verbessern.

Prof. em. Dr. med. Dr. h. c. D. Reinhardt

\section{Positives Bronchopneumogramm zeigt Infektion an}

\begin{abstract}
Eine 26-jährige Frau mit terminaler Niereninsuffizienz aufgrund einer Lupusnephritis stellte sich wegen Fieber, Husten, Atemnot und weiteren Pneumonie-verdächtigen Symptomen vor. Die Symptomatik bestand seit drei Tagen. Als sich ein akutes Lungenversagen eingestellt hatte, benötigte die Patientin schließlich eine extrakorporale Membranoxygenierung (ECMO), nachdem eine mechanische Ventilation ineffizient war. Ein doppellumiger ECMO-Katheter wurde in der rechten V. jugularis interna platziert, der Blut aus der V. cava superior und inferior ableitete und über den das Blut in den rechten Vorhof zurückströmte. Die Bettaufnahme des Thorax zeigte eine bilaterale Verschattung und ein Bronchopneumogramm. Dieses radiologische Zeichen entsteht, wenn luftgefüllte Bronchien als Folge einer Verschattung der umgebenden Alveolen deutlich sichtbar werden. In Kulturen der bronchoalveolären Lavage wuchsen Moraxella catarrhalis und Hämophilus Influenza.

Die Patientin wurde antibiotisch behandelt und dialysiert. Am neunten Krankheitstag konnte die ECMO beendet werden. Die Patientin wurde in eine Reha-Einrichtung überwiesen. Bei einer Nachuntersuchung sechs Monate später ging es ihr sehr gut.
\end{abstract}

Prof. Dr. med. H. S. FüeßI

- Natt B, Raz Y (bnatt@deptofmed.arizona.edu). Air Bronchogram. NEnglJ Med. 2015;373:2663

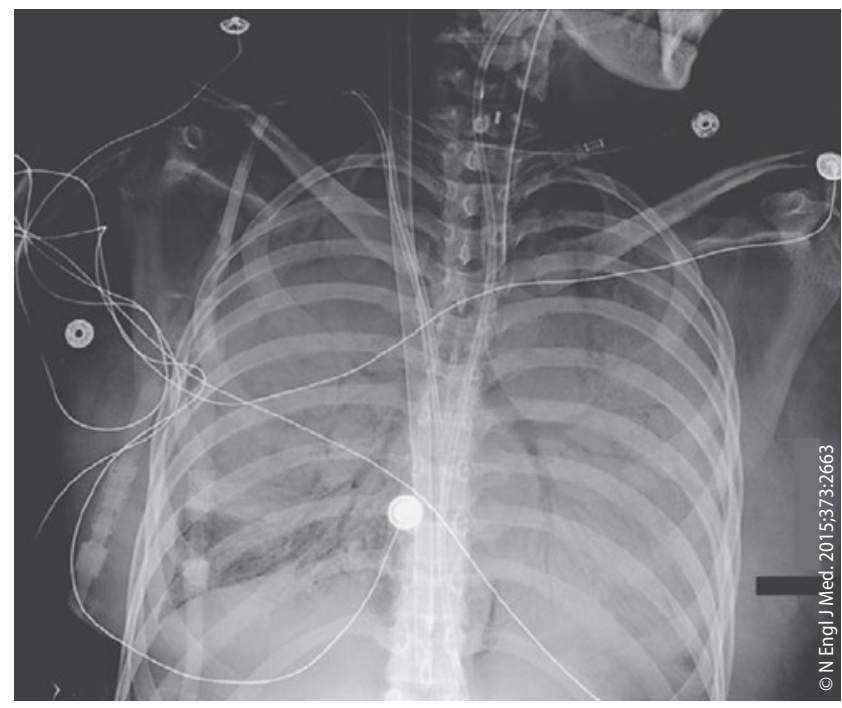

Bronchopneumogramm: deutlich sichtbare, luftgefüllte Bronchien als Folge einer Verschattung der umgebenden Alveolen. 